\title{
ANTICONVULSANT SUPPRESSION OF POSTNATAL NEUROGENESIS IN LABORATORY ANIMALS
}

The effects of phenobarbital and diazepam on cell proliferation and neurogenesis were studied in newborn rats followed for 6 months, in a study at University of Dresden, Germany; Medical University, Lublin, Poland; University Medicine Berlin, Germany; and Solvay Research Laboratories, Weesp, The Netherlands. The N-methyl-D-aspartate antagonist MK801, and the GABA subtype A agonists phenobarbital and diazepam administered to infant rats on postnatal days 6-10 caused reduced numbers of neurons in the hippocampal dentate gyrus at postnatal day 15. No apoptosis was demonstrated. At age 6 months, phenobarbital-treated rats had fewer neurons in the dentate gyrus and performed worse than saline-treated littermates in water maze learning and memory task. Blockade of $\mathrm{N}$-methyl-D-aspartate receptor-mediated excitation and enhancement of GABA subtype A receptor activation impair cell proliferation and inhibit neurogenesis in the immature rat brain. These findings raise concerns about the frequent use of phenobarbital in the treatment of neonatal seizures. (Stefovska VG, Uckermann O, Czuczwar M, et al. Sedative and anticonvulsant drugs suppress postnatal neurogenesis. Ann Neurol Oct 2008;64:434-445). (Respond: Dr Ikonomidou, Department of Pediatric Neurology, Children's Hospital, Medical Faculty Carl Gustav Carus, University of Technology Dresden, Fetscherstrasse 74, 01307 Dresden, Germany).

COMMENT. Neurogenesis in the hippocampal dentate gyrus is at its peak during the first week of postnatal life and declines progressively after day 9 in newborn rats. Phenobarbital administered in the first week to 10 days results in reduced neurogenesis at 2 weeks postnatally and impairment of learning and memory at 6 months, equivalent to adult life. These effects result in decreased hippocampal volume, reduced neuronal densities in the dentate gyrus, the CA1-hippocampus, and the cingulate cortex. These observations call for caution regarding the use of NMDA receptor antagonists and GABA-a agonists in neonatal, pediatric, and obstetric medicine.

\section{SLEEP DISORDERS}

\section{SLEEP TERRORS IN TWINS}

In an attempt to clarify the genetic and environmental causes of sleep terrors in childhood, reasearchers in Canada followed 390 pairs of monozygotic and dizygotic twins by assessing the frequency of sleep terrors at 18 and 30 months of age using a questionnaire administered to the biological mothers. The prevalence of sleep terrors was $36.9 \%$ at 18 months and $19.7 \%$ at 30 months. Boys and girls were affected equally. The polychoric correlations were 0.63 monozygotic and 0.36 dizygotic at 18 months and 0.68 and 0.24 at 30 months. Sleep terrors were best explained by a genetic and non-shared environmental, 2component model. At 18 months, genetic factors accounted for $43.7 \%$ and non-shared environmental factors for $56.3 \%$ of the phenotypic variance; at 30 months, these proportions were $41.5 \%$ and $58.5 \%$, respectively. (Nguyen BH, Perusse D, Paquet J, et al. Sleep terrors in children: a prospective study of twins. Pediatrics Dec 2008; 122:e1164-e1167). (Respond: 
Jacques Montplaisir MD, PhD, Sleep Disorders Center, Sacre-Coeur Hospital, Montreal, Quebec, Canada H4J 1C5).

COMMNT. The prevalence of sleep terrors is high in infants (37\% at 18 months) and decreases by one half to approximately $20 \%$ by 30 months of age. Genetic factors play an important role in the etiology of this early childhood parasomnia, accounting for $>40 \%$ of the phenotypic variance for both 18 - and 30-month-old twins. The role of non-shared environmental factors is also significant, $>55 \%$ of the variance at both 18 and 30 months. Night terrors have a combination genetic-environmental etiology, but to date, no specific genes have been identified.

\section{GENETICALLY DETERMINED EEG FINGERPRINT OF SLEEP}

The influence of genetic factors on the individual profile of sleep electroencephalographic (EEG) power spectra at the 8 to $16 \mathrm{~Hz}$ frequency range during nonrapid eye movement (NREM) sleep was determined by recording 40 monozygotic and dizygotic twins during sleep. The study performed at the University of Rome and various international centers found that this EEG fingerprint of sleep showed a greater similarity in monozygotic than dizygotic pairs, with a $96 \%$ estimate of heritability. (De Gennaro L, Marzano C, Fratello F, et al. The electroencephalographic fingerprint of sleep is genetically determined: a twin study. Ann Neurol Oct 2008;64:455-460). (Respond: Dr De Gennaro, Department of Psychology, Section of Neuroscience, University of Rome "Sapienza," Via dei Marsi, 78, 00185 Rome, Italy. E-mail: luigi.degennaro(a)uniromal.it).

COMMENT. Healthy humans have a unique profile of the sleep electroencephalographic (EEG) power spectra at the 8 to $16 \mathrm{~Hz}$ frequency range duing nonrapid eye movement (NREM) sleep. This fingerprint allows discrimination between individuals with a probability of $92 \%$ (De Gennaro L et al, 2005). These authors have shown that individual differences in this EEG fingerprint of NREM sleep are genetically determined. A genetic contribution has already been demonstrated for the awake-resting EEG alpha power, and also, for many sleep disorders, including night terrors, narcolepsy, obstructive sleep apnea, restless legs syndome, and Kleine-Levin syndrome.

\section{NEUROMUSCULAR DISORDERS}

\section{HAND INVOLVEMENT IN CHARCOT-MARIE-TOOTH DISEASE 1A}

Hand strength, function and disease-related symptoms were determined in 84 children, aged 2-16 years, with Charcot-Marie-Tooth disease type 1A (CMT1A) at University of Sydney, Children's Hospital at Westmead, and Royal Children's Hospital, Parkville, Australia. Hand weakness and dysfunction was present from the earliest stages of the disease and tended to worsen with age throughout childhood. Poor handwriting, weakness, pain and sensory symptoms also worsened with age. (Burns J, Bray P, Cross LA, North KN, Ryan MM, Ouvrier RA. Hand involvement in children with Charcot-Marie-Tooth disease type 1A. Neuromuscul Disord Dec 2008;18:970-973). (Respond: Dr Joshua Burns, 